\title{
Uso del ultrasonido terapéutico pulsado en el tratamiento de personas con osteoartritis de rodilla
}

\author{
Pulsed therapeutic ultrasound in the treatment \\ of persons with knee osteoarthritis
}

\author{
Eliana Isabel Rodríguez Grande ${ }^{1,2}$, Liliana Carolina Ramírez Ramírez²
}

Forma de citar: Rodríguez Grande EI, Ramírez Ramírez LC. Uso del ultrasonido terapéutico pulsado en el tratamiento de personas con osteoartritis de rodilla. Rev Univ Ind Santander Salud. 2015; 47(3): 337-348. DOI: http://dx.doi.org/10.18273/revsal.v47n3-2015010 @) (1) Ð

\section{RESUMEN}

La osteoartritis de rodilla es una patología crónica y degenerativa caracterizada por la destrucción del cartílago articular, para la cual en la actualidad no existen intervenciones terapéuticas que detengan el deterioro. Una de las modalidades utilizadas en fisioterapia para el tratamiento de la osteoartritis es el ultrasonido terapéutico, sin embargo los resultados reportados en la literatura sobre el efecto del ultrasonido terapéutico en pacientes con osteoartritis de rodilla son contradictorios y limitan la toma de decisiones en el ámbito clínico. En este sentido, en esta revisión se realizó el análisis crítico de la evidencia científica que evalúa el efecto del ultrasonido en pacientes con OA de rodilla. Fue realizado un acercamiento a los parámetros adecuados para alcanzar efectos terapéuticos, además se analizaron los posibles mecanismos por los cuales el ultrasonido terapéutico puede favorecer dichos efectos.

Palabras clave: Dolor, rehabilitación, terapia por ultrasonido, fisioterapia, modalidades de fisioterapia, osteoartritis de rodilla.

\begin{abstract}
Knee osteoarthritis is a chronic, degenerative disease without therapeutic interventions toward prevent the articular cartilage deterioration, thus pain and functionality limitation are the main goals of the physical therapy treatment. The therapeutic ultrasound is a common physical modality used for the treatment of osteoarthritis, however, the results reported in the literature about the therapeutic effect of this physical modality in patients with knee osteoarthritis are contradictory and limits clinical decision making. Therefore, we made a critical analysis of scientific evidence about this matter, we made an approached to the appropriate parameters to achieve therapeutic effects and we analyzed the possible mechanisms by which these effects can occur.
\end{abstract}

Keywords: Pain, rehabilitation, ultrasound therapy, physiotherapy, physical modalities.

1. Universidad del Rosario. Bogotá D.C., Colombia

2. Universidad Industrial de Santander. Bucaramanga, Colombia

Correspondencia: Eliana Isabel Rodríguez Grande. Dirección: Carrera 24 N 63D-69, Bogotá D.C. Correo electrónico: eliana. rodriguez@urosario.edu.co. Teléfono: +1 2970200 ext.3420. 


\section{INTRODUCCIÓN}

La osteoartritis (OA), es una patología crónica, degenerativa caracterizada por el desgaste del cartílago articular cuyas principales manifestaciones clínicas son dolor, disminución de la movilidad articular y de la fuerza muscular, rigidez, además de la efusión articular en estadios avanzados de la enfermedad ${ }^{1}$.

La OA es considerada la principal causa de dolor músculo esquelético y discapacidad en los adultos mayores en el mundo siendo una de las condiciones que más impacto tiene sobre la funcionalidad de los adultos mayores $^{1,2}$. En los Estados Unidos afecta al $12.1 \%$ de la población adulta y compromete principalmente a mujeres, quienes tienen un riesgo relativo de 1.79 , la articulación de soporte de peso corporal que presenta mayor incidencia es la rodilla, con una incidencia de 240 casos nuevos por cada 100.000 personas-año en comparación con la OA de cadera cuya incidencia es de 88 casos nuevos por 100.000 personas-año ${ }^{2}$.

En Colombia los años de vida saludables perdidos en forma prematura por discapacidad (AVISA) a causa de la OA son similares a los AVISA perdidos por cáncer de próstata y cáncer de cérvix, por lo cual la OA puede ser considerada un problema de salud pública ${ }^{3}$.

El dolor es la principal causa de limitación funcional en personas con OA de rodilla. Está presente entre el 38 y el $68 \%{ }^{2}$ de las personas que sufren la enfermedad, genera un impacto negativo sobre la fuerza muscular y el rango de movimiento articular, lo cual a su vez lleva al menoscabo progresivo de la funcionalidad y por ende de la calidad de vida de estas personas ${ }^{2}$.

El tratamiento de las personas con $\mathrm{OA}$ incluye principalmente intervenciones de tipo farmacológico a través de antiinflamatorios no esteroideos (AINES) y manejo no farmacológico'. Las guías ACR 2012 (American College of Reumatology $y^{4}$ ) para el tratamiento de la OA recomiendan el tratamiento no farmacológico, incluyendo la terapia física, como estrategia de primera de línea en pacientes con OA, cuya aplicación contribuye a disminuir el dolor y a mejorar la funcionalidad ${ }^{5}$.

Una de las modalidades terapéuticas ampliamente utilizadas en fisioterapia para el tratamiento de la OA es el ultrasonido terapéutico (UST) ${ }^{6}$. Se ha planteado que el UST podría disminuir la intensidad del dolor, mejorando el rango de movimiento articular y la fuerza muscular a través de la disminución de inhibición de la vía $\mathrm{Ib}$ dada la disminución de las aferencias de los mecanorreceptores de Paccini y las terminaciones nerviosas libres ${ }^{7}$, aumentado así la funcionalidad de las personas con OA de rodilla ${ }^{8}$. Sin embargo, los resultados reportados en la literatura sobre el efecto del UST en pacientes con OA de rodilla son contradictorios y las limitaciones metodológicas presentes en los mismos, dificultan la toma de decisiones en el ámbito clínico con base en los estudios relacionados con este tema.

Esta revisión tiene por objetivo analizar críticamente de la evidencia científica disponible sobre el efecto del UST en el tratamiento de personas con OA de rodilla, aproximarse a los parámetros adecuados para alcanzar efectos terapéuticos y analizar los posibles mecanismos por los cuales el UST puede favorecer este efecto, teniendo en cuenta que estos dos últimos temas no han sido abordados a profundidad por las revisiones previas disponibles en la literatura ${ }^{5,6}$.

\section{MÉTODOS DE BÚSQUEDA}

Se realizó la búsqueda de artículos publicados hasta octubre de 2014 en las bases de datos PUBMED, MEDLINE, PEDro, CINAHL y EMBASE.

Las palabras claves utilizadas para la búsqueda electrónica fueron "Therapeutic ultrasound", "knee osteoarthritis", "physical therapy", "physiotherapy treatment", "Physical agents" and "Pulsed ultrasound". La búsqueda se limitó a estudios realizados en humanos que incluyeran al menos la evaluación de la intensidad del dolor y la funcionalidad, las cuales son importantes de ser evaluadas en personas con OA de rodilla, según el grupo de medidas de resultado en ensayos clínicos controlados (ECC) en reumatología OMERACT (Outcome Measures in Rheumatology $y^{9}$. También se tuvieron en cuenta estudios que incluyeran otras medidas de resultado relacionadas con los objetivos de tratamiento fisioterapéutico como la movilidad articular y la fuerza muscular ${ }^{10}$.

\section{CRITERIOS DE ELEGIBILIDAD DE LOS ESTUDIOS}

Se incluyeron ECC en idioma español o inglés cuya población de estudio fueran adultos (18 años o más) con diagnóstico de OA de rodilla confirmada por criterios clínicos y/o radiográficos, en los cuales se evaluara el efecto terapéutico del UST. Adicionalmente, solo se incluyeron en la revisión aquellos artículos que presentaran como parámetros de aplicación al menos la 
forma de generación del UST, la intensidad, el tiempo de aplicación y la ERA (Efective Radiating Area) o el tamaño del cabezal.

\section{RESULTADOS EN LA BÚSQUEDA BIBLIOGRÁFICA}

Veinticinco estudios cumplieron con los criterios de búsqueda, de los cuales fueron descartados 2 por encontrarse en otro idioma diferente al inglés o español, 7 por no estar disponible el contenido completo y 2 por no incluir los parámetros de aplicación del UST. Así, la búsqueda bibliográfica concluyó con $13 \mathrm{ECC}^{11-23}$ (figura 1). La tabla 1 muestra los artículos incluidos en la revisión describiendo el objetivo, la población del estudio, las variables, el análisis y los principales resultados. Así mismo en la tabla 2 se describe el diseño metodológico de cada uno de ellos.
- 7 No disponible en texto completo

- 2 en otro idioma diferente al inglés

- 2 excluidos por no incluir los parámetros de aplicación.
Búsqueda en bases de datos

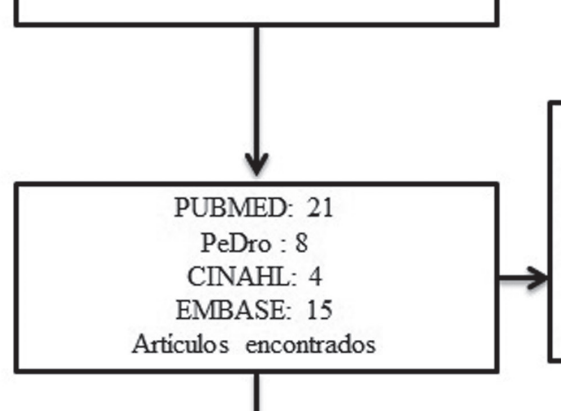

- 3 Ultrasonido diagnóstico

- 4 Otras modalidades de tratamiento.

- 6 UST en otras patologías.

- 4 experimentales en modelo animal.

2 experimentales in vitro

4 Revisiones y metaanálisis

1 estudio cuasiexperimental

13 estudios cumplieron criterios de

elegibilidad

FIGURA 1. Flujograma de los estudios incluidos en la revisión.

Los artículos incluidos en la revisión fueron sometidos a la evaluación de su calidad metodológica con la escala de PeDro, la cual incluye diez items sobre la validez interna y el análisis estadístico, asignando un puntaje entre 0 y $10^{24}$. Fueron incluidos los estudios que alcanzaron mínimo 5 puntos en la escala de PeDro. Dentro de la evaluación de la calidad metodológica, se definió que estudios con puntajes entre 7 y 10 eran considerados de alta calidad metodológica, entre 5 y 6 de calidad intermedia y entre 0 y 4 de baja calidad metodológica (Tabla 2).

Los puntajes de los estudios incluidos estuvieron en los rangos de 5-10, vale la pena resaltar que en 11 de los 13 estudios no se tuvo en cuenta el enmascaramiento del participante y del terapeuta tratante. El puntaje máximo fue de 10 en dos de los estudios ${ }^{12,23}$ y el puntaje mínimo fue de 5 para el estudio de Kozanoglu et al. ${ }^{17}$ (Tabla 2). Dentro de las limitaciones metodológicas encontradas en los estudios revisados se destaca principalmente la ausencia de un cálculo de tamaño muestral, lo que puede afectar el poder del estudio para establecer diferencias induciendo al error sistemático tipo II.

Solo dos de los estudios revisados ${ }^{12,13}$, reportan el cálculo del tamaño de muestra, pero no es clara la forma como este fue realizado.

Por otro lado, las variables explicatorias no son de fácil comparación, pues el UST es aplicado junto con otras modalidades de intervención sin permitir distinguir los efectos de esta modalidad de los efectos de otras modalidades aplicadas. Cetin et al. ${ }^{14}$ evaluaron los efectos terapéuticos de agentes físicos como la diatermia, el TENS y UST mientras que Huang et al. ${ }^{13}$ estudiaron los efectos del uso conjunto de intervenciones como las inyecciones de ácido hialurónico, UST y fortalecimiento isocinético aplicadas en conjunto. 
TABLA 1. Descripción de los ensayos clínicos controlados incluidos en la revisión

\begin{tabular}{|c|c|c|c|c|}
\hline Autor-año & Objetivo & $\begin{array}{l}\text { Población de } \\
\text { Estudio }\end{array}$ & Variables & Resultados \\
\hline $\begin{array}{l}\text { Kozanoglu E } \\
\text { et al. } 2003\end{array}$ & $\begin{array}{l}\text { Comparar la eficacia de la } \\
\text { fonoforesis con ibuprofeno } \\
\text { y el UST }\end{array}$ & $\begin{array}{c}\mathrm{n}=60 \\
\text { Mujeres: } 51 \\
\text { Hombres: } 9 \\
\text { Edad= } 59.8 \pm 9.9\end{array}$ & $\begin{array}{l}\text { Salida } \\
\text { WOMAC, dolor, rango de } \\
\text { movimiento, funcionalidad } \\
\text { Explicatorias } \\
\text { G1. UST, G2. UST y } 5 \% \text { de } \\
\text { ibuprofeno }\end{array}$ & $\begin{array}{l}\text { No se encontraron } \\
\text { significativas }\end{array}$ \\
\hline $\begin{array}{l}\text { Huang M } \\
\text { et al. } \\
2005\end{array}$ & $\begin{array}{l}\text { Investigar } \\
\text { efecto del UST en } \\
\text { un programa de ejercicios } \\
\text { de fortalecimiento muscular } \\
\text { isocinético en el estado } \\
\text { funcional }\end{array}$ & $\begin{array}{c}\mathrm{n}=120 \\
\begin{array}{c}\text { Mujeres razón } 4.2: 1 \\
\text { hombre }\end{array} \\
\text { Edad }=62.0 \pm 8.4\end{array}$ & $\begin{array}{l}\text { Salida } \\
\text { Intensidad dolor, funcionalidad, } \\
\text { fuerza muscular, movilidad } \\
\text { articular, discapacidad. } \\
\text { Explicatorias } \\
\text { G1: FId, G2: FI y UST continuo, } \\
\text { G3:FIy UST pulsado, G4:Control }\end{array}$ & $\begin{array}{l}\text { Dolor: } 5.0 \pm 1.3,2.6 \pm 1.7 \\
\text { Funcionalidad: } 73.2 \pm 6.0,92.4 \pm 3.4 \\
\text { Fuerza: } 493.1,653.3 \\
\text { Discapacidad: } 7.1 \pm 2.1,4.1 \pm 0.6 \\
\text { Movilidad articular: } 105 \pm 12,119 \pm 15\end{array}$ \\
\hline $\begin{array}{l}\text { Huang M } \\
\text { et al. } \\
2005\end{array}$ & $\begin{array}{l}\text { Investigar los efectos de } \\
\text { la terapia integrada en el } \\
\text { estado funcional }\end{array}$ & $\begin{array}{c}\quad \mathrm{n}=140 \\
\text { Mujeres: } 113 \\
\text { Hombres: } 27 \\
\text { Edad }=40-77\end{array}$ & $\begin{array}{l}\text { Salida } \\
\text { Intensidad dolor, funcionalidad, } \\
\text { fuerza muscular, discapacidad, } \\
\text { movilidad articular, goniometría } \\
\text { Explicatorias } \\
\text { G1: FI, G2:FI y UST pulsado, } \\
\text { G3: FI y UST pulsado y ácido } \\
\text { hialurónico, G4: Control }\end{array}$ & $\begin{array}{l}\text { Diferencias significativas entre } \mathrm{G} 2 \text { y G3 } \\
\text { Dolor: } 5.5 \pm 1.7,3.0 \pm 1.8 \\
\text { Funcionalidad: } 71.3 \pm 6.7,90.2 \pm 3.1 \\
\text { Fuerza: } 480.1,683.3 \\
\text { Discapacidad: } 7.4 \pm 1.6,4.4 \pm 1.1 \\
\text { Movilidad articular: } 104 \pm 10,114 \pm 15\end{array}$ \\
\hline $\begin{array}{l}\text { Cetin N } \\
\text { et al. } \\
2008\end{array}$ & $\begin{array}{l}\text { Investigar los efectos } \\
\text { terapéuticos de los agentes } \\
\text { físicos administrados antes } \\
\text { del ejercicio isocinético en } \\
\text { mujeres con OA de rodilla. }\end{array}$ & $\begin{array}{c}\mathrm{n}=100 \\
\text { Mujeres: } 100 \\
\text { OA bilateral } \\
\text { Edad }=59.82 \pm 9.1\end{array}$ & $\begin{array}{l}\text { Salida } \\
\text { Discapacidad, dolor, fuerza } \\
\text { muscular, funcionalidad. } \\
\text { Explicatorias } \\
\text { G1: diatermia de onda corta, } \\
\text { calor y FI, G2:TENS, calor y FI, } \\
\text { G3: UST, calor local y FI, G4: } \\
\text { calor local y FI, G5: FI. }\end{array}$ & $\begin{array}{l}\text { Discapacidad: } 11.40 \pm 2.45,7.67 \pm 2.30 \\
\text { Dolor: } 5.90 \pm 1.45,3.55 \pm 1.41 \\
\text { Fuerza. } 36.00 \pm 8.32,52.35 \pm 10.89 \\
\text { Funcionalidad: } 53.10 \pm 9.84,42.60 \pm 11.50\end{array}$ \\
\hline
\end{tabular}

\begin{tabular}{|c|c|c|c|c|}
\hline Autor-año & Objetivo & $\begin{array}{l}\text { Población de } \\
\text { Estudio }\end{array}$ & Variables & Resultados \\
\hline $\begin{array}{l}\text { Özgönenel L } \\
\text { et al. } \\
2009\end{array}$ & $\begin{array}{l}\text { Determinar la efectividad } \\
\text { del UST en OA de rodilla }\end{array}$ & $\begin{array}{c}\mathrm{n}=67 \\
\text { Mujeres:54 } \\
\text { Hombres: } 13 \\
\text { Edad }=54.8 \pm 7\end{array}$ & $\begin{array}{l}\text { Salida } \\
\text { Dolor, PE, Funcionalidad } \\
\text { Explicatorias } \\
\text { G1: UST continuo, G2: Placebo }\end{array}$ & El UST no superó al grupo placebo. \\
\hline $\begin{array}{l}\text { Tascioglu F } \\
\text { et al. } \\
2010\end{array}$ & $\begin{array}{l}\text { Investigar la eficacia del } \\
\text { UST a corto plazo en } \\
\text { pacientes con OA de rodilla. }\end{array}$ & $\begin{array}{c}\mathrm{n}=90 \\
\text { Mujeres:59 } \\
\text { Hombres: } 31 \\
\text { Edad }=61.6 \pm 3.7\end{array}$ & $\begin{array}{l}\text { Salida } \\
\text { Dolor, PE, WOMAC, movilidad } \\
\text { articular, Funcionalidad } \\
\text { Explicatorias } \\
\text { G1: UST continuo, G2: UST } \\
\text { pulsado, Grupo 3: placebo }\end{array}$ & $\begin{array}{l}\text { Dolor: } 6.89 \pm 1.39,5.25 \pm 1.90 \\
\text { PE: } 43.43 \pm 8.26,35.61 \pm 8.73 \\
\text { Movilidad articular: } 35.61 \pm 8.73 \\
\text { Funcionalidad: } 22.57 \pm 2.08,20.00 \pm 1.94\end{array}$ \\
\hline $\begin{array}{l}\text { Mascarin N } \\
\text { et al. } \\
2012\end{array}$ & $\begin{array}{llr}\text { Investigar } & \text { los } & \text { efectos } \\
\text { de } & \text { kinesioterapia } \\
\text { y electroterapia en la } \\
\text { capacidad de ejercicio } \\
\text { funcional }\end{array}$ & $\begin{array}{c}\mathrm{n}=40 \\
\text { Mujeres: } 40 \\
\text { Edad }=62.8 \pm 7.6\end{array}$ & $\begin{array}{l}\text { Salida } \\
\text { Funcionalidad, rango de } \\
\text { movimiento, intensidad de dolor, } \\
\text { PE } \\
\text { Explicatorias } \\
\text { G1. kinesioterapia, G2. TENS, } \\
\text { G3. } \\
\text { UST }\end{array}$ & $\begin{array}{l}\text { Funcionalidad: } 318 \pm 68,358 \pm 77 \\
\text { Dolor: } 6.6 \pm 3.0,4.5 \pm 3.7 \\
\text { PE: } 53.5 \pm 12.2,28.8 \pm 14.8\end{array}$ \\
\hline $\begin{array}{l}\text { Ulus Y } \\
\text { et al. } \\
2012\end{array}$ & $\begin{array}{l}\text { Evaluar la efectividad del } \\
\text { UST en pacientes con OA } \\
\text { de rodilla. }\end{array}$ & $\begin{array}{c}\mathrm{n}=42 \\
\text { Mujeres: } 34 \\
\text { Hombres: } 6 \\
\text { Edad }=60.25 \pm 8.8\end{array}$ & $\begin{array}{l}\text { Salida } \\
\text { Dolor, PE, funcionalidad } \\
\text { Explicatorias } \\
\text { G1:UST continuo, G2: placebo }\end{array}$ & $\begin{array}{l}\text { Diferencias significativas en ambos } \\
\text { grupos. El UST no superó al grupo } \\
\text { placebo }\end{array}$ \\
\hline $\begin{array}{l}\text { Loyola A } \\
\text { et al. } \\
2012\end{array}$ & $\begin{array}{l}\text { Evaluar la efectividad del } \\
\text { UST en pacientes con OA } \\
\text { de rodilla. }\end{array}$ & $\begin{array}{c}\mathrm{n}=10 \\
\text { Mujeres: } 9 \\
\text { Hombres: } 1 \\
\text { Edad }=56-81\end{array}$ & $\begin{array}{l}\text { Salida } \\
\text { Dolor, funcionalidad } \\
\text { Explicatorias } \\
\text { G1:UST continuo, G2: placebo }\end{array}$ & $\begin{array}{l}\text { Dolor: } 7.1 \pm 2.5,4.2 \pm 2.2 \\
\text { Funcionalidad: } 10.5 \pm 5.4,5 \pm 4.5\end{array}$ \\
\hline
\end{tabular}




\begin{tabular}{|c|c|c|c|c|}
\hline Autor-año & Objetivo & $\begin{array}{l}\text { Población de } \\
\text { Estudio }\end{array}$ & Variables & Resultados \\
\hline $\begin{array}{l}\text { Luksurapan } \\
\text { W et al. } \\
2013\end{array}$ & $\begin{array}{l}\text { Comparar los efectos de piroxican } \\
\text { y el UST en OA sintomática de } \\
\text { rodilla. }\end{array}$ & $\begin{array}{c}\mathrm{n}: 46 \\
\text { mujeres: } 45 \\
\text { hombre: } 1 \\
\text { Edad }=58.9 \pm 10.5\end{array}$ & $\begin{array}{l}\text { Salida } \\
\text { Dolor, PE } \\
\text { Explicatorias: } \\
\text { G1: UST y piroxican, G2: } \\
\text { UST }\end{array}$ & $\begin{array}{l}\text { Dolor G1: } 70.6 \pm 12.0,23.6 \pm 19.3 \\
\text { Dolor G2: } 72.5 \pm 12.3,43.9 \pm 25.2 \\
\text { PE G1: } 108.4 \pm 32.5,50.4 \pm 31.7 \\
\text { PE G2: } 128.2 \pm 31.0,89.0 \pm 50.3\end{array}$ \\
\hline $\begin{array}{l}\text { Boyaci A et } \\
\text { al. } 2013\end{array}$ & $\begin{array}{l}\text { Comparación de la eficacia de } \\
\text { fonoforesis de ketoprofeno, } \\
\text { UST, y la diatermia de onda corta } \\
\text { en la OA de rodilla }\end{array}$ & $\begin{array}{c}\mathrm{n}: 101 \\
101 \text { mujeres } \\
\text { Edad }=52.5 \pm 4.8\end{array}$ & $\begin{array}{l}\text { Salida } \\
\text { Dolor, PE, funcionalidad. } \\
\text { Explicatorias } \\
\text { G1: Fonoforesis, G2: UST } \\
\text { continuo, G3: Diatermia de } \\
\text { onda corta }\end{array}$ & $\begin{array}{l}\text { Dolor: } 7.7 \pm 2.1,5.8 \pm 2.0 \\
\text { PE: } 55.1 \pm 16.8,44.2 \pm 15.3 \\
\text { Funcionalidad: } 22.9 \pm 4.7\end{array}$ \\
\hline $\begin{array}{l}\text { Cakir S et al. } \\
2014\end{array}$ & $\begin{array}{l}\text { Comparar la efectividad del UST } \\
\text { continuo, pulsado y placebo en } \\
\text { OA de rodilla. }\end{array}$ & $\begin{array}{c}\mathrm{n}: 60 \\
\text { mujeres: } 47 \\
\text { hombres: } 13 \\
\text { Edad }=58.2 \pm 9.9\end{array}$ & $\begin{array}{l}\text { Salida } \\
\text { Dolor, funcionalidad, PE } \\
\text { Explicatorias } \\
\text { G1: UST continuo, G2: UST } \\
\text { pulsado, G3: UST placebo }\end{array}$ & $\begin{array}{l}\text { Diferencias en todos los grupos, no entre } \\
\text { grupos. }\end{array}$ \\
\hline $\begin{array}{l}\text { Oktayoğlu P } \\
2014\end{array}$ & $\begin{array}{l}\text { Comparar la eficacia de } \\
\text { fonoforesis y UST en personas } \\
\text { con OA primaria de rodilla. }\end{array}$ & $\begin{array}{c}\mathrm{n}: 40 \\
\text { mujeres: } 30 \\
\text { hombres: } 10 \\
\text { Edad }=54.5 \pm 8.7\end{array}$ & $\begin{array}{l}\text { Salida } \\
\text { Dolor, PE, } \\
\text { Explicatorias } \\
\text { G1: Fonoforesis, G2: UST } \\
\text { continuo }\end{array}$ & $\begin{array}{l}\text { Dolor reposo: } 48 \pm 22.1,40 \pm 17.7 \\
\text { PE: } 15.9 \pm 3.9,12.6 \pm 2.0\end{array}$ \\
\hline
\end{tabular}

UST: Ultrasonido terapéutico; n: tamaño de muestra; G: Grupo de intervención; FI: fortalecimiento isocinético; PE: percepción de la enfermedad; WOMAC: The Western Ontario and McMaster Universities questionnaire; TENS: Estimulador Eléctrico Transcutáneo.

TABLA 2. Análisis de la calidad metodológica de los estudios.

\begin{tabular}{lcccccccccccc}
\hline Estudio & $\mathbf{1}$ & $\mathbf{2}$ & $\mathbf{3}$ & $\mathbf{4}$ & $\mathbf{5}$ & $\mathbf{6}$ & $\mathbf{7}$ & $\mathbf{8}$ & $\mathbf{9}$ & $\mathbf{1 0}$ & $\mathbf{1 1}$ & Puntaje total \\
\hline Kozanoglu E 2003 & 1 & 0 & 0 & 1 & 0 & 0 & 0 & 1 & 1 & 1 & 1 & 5 \\
Huang M 2005 & 1 & 1 & 1 & 1 & 0 & 0 & 0 & 1 & 1 & 1 & 1 & 7 \\
Huang M 2005 & 1 & 1 & 1 & 1 & 0 & 0 & 1 & 1 & 1 & 1 & 1 & 8 \\
Cetin N 2008 & 1 & 1 & 0 & 1 & 0 & 0 & 1 & 1 & 1 & 1 & 1 & 7 \\
Özgönenel L 2009 & 1 & 1 & 1 & 1 & 0 & 0 & 1 & 1 & 1 & 1 & 1 & 8 \\
Tascioglu F 2010 & 1 & 1 & 1 & 1 & 0 & 0 & 1 & 1 & 1 & 1 & 1 & 8 \\
Mascarin N 2012 & 1 & 1 & 1 & 1 & 1 & 0 & 0 & 1 & 1 & 1 & 1 & 8 \\
Ulus Y 2012 & 1 & 1 & 1 & 1 & 0 & 0 & 1 & 1 & 1 & 1 & 1 & 8 \\
Loyola A 2012 & 1 & 1 & 1 & 1 & 1 & 1 & 1 & 1 & 1 & 1 & 1 & 10 \\
Luksurapan W 2013 & 1 & 1 & 1 & 1 & 1 & 1 & 1 & 1 & 1 & 1 & 1 & 10 \\
Boyaci A 2013 & 1 & 1 & 1 & 1 & 0 & 0 & 1 & 1 & 1 & 1 & 1 & 8 \\
Cakir S 2014 & 1 & 1 & 1 & 1 & 1 & 0 & 1 & 1 & 1 & 1 & 1 & 9 \\
Oktayoğlu P.2014 & 1 & 1 & 1 & 1 & 0 & 0 & 0 & 1 & 1 & 1 & 1 & 7 \\
\hline
\end{tabular}

Ítems de la escala de PEDro: 1. Criterios de elegibilidad; 2. Asignación aleatoria; 3. Enmascaramiento de la asignación; 4. Similitud al inicio del estudio; 5. Enmascaramiento del participante; 6. Enmascaramiento del terapeuta; 7. Enmascaramiento del evaluador; 8. Mínimo 85\% de seguimiento; 9. Análisis por intención de tratar; 10. Comparación estadística entre grupos; 11. Medidas puntuales y de variabilidad. Ítem 1 No se incluye en la puntuación de la escala PEDro.

En el mismo sentido, las variables de salida evaluadas no son comparables entre los estudios. Cetin et al. ${ }^{14}$ evaluaron los efectos del UST sobre el dolor, la funcionalidad y la fuerza muscular; Mascarin et al. ${ }^{15}$, evaluaron velocidad de marcha y funcionalidad con un instrumento diferente al utilizado por Cetin et al. ${ }^{14}$, Huang et al. ${ }^{11,13}$ determinaron como variables de salida la intensidad del dolor, el pico de toque y la funcionalidad mientras que, Loyola et al. ${ }^{23}$ estudiaron sí la aplicación del UST produce efectos positivos en la regeneración del cartílago articular. 
Los estudios revisados presentan sesgos de selección, no aleatorización ${ }^{17}$ y falta de homogeneidad de los grupos al inicio del estudio ${ }^{11,13}$; sesgos de clasificación (falta de enmascaramiento ${ }^{14,17}$ y el uso de instrumentos sin evaluación de propiedades psicométricas ${ }^{23}$ ), sesgos de confusión (ninguno estudio estableció el análisis de los resultados teniendo en cuenta las variables de confusión). Además en algunos estudios hubo factores de confusión como en el trabajo de Cetin et al. ${ }^{14}$ quienes incluyeron la aplicación de calor superficial a través de un paquete caliente en todos los grupos de intervención, excepto en el grupo placebo, lo cual pudo influir en los resultados de la intervención.

Otra de las características de los estudios que dificulta la comparación de los mismos es el número de intervenciones y los resultados contradictorios. Cetin et al. ${ }^{14}$ aplicaron UST durante 24 sesiones, mostrando diferencias significativas en las variables de salida en todos los grupos en comparación con el placebo; Ozgonenel et al. ${ }^{19}$ realizaron 10 sesiones de intervención sin encontrar diferencias significativas entre el grupo de intervención y el placebo. Esto pudo estar influenciado por la falta de poder de este estudio debido a las pérdidas y exclusiones luego de la aleatorización. Ulus et al. ${ }^{18}$ realizaron 15 sesiones de intervención en un grupo de UST continuo y otro placebo sin encontrar diferencias significativas entre los grupos.

A continuación se presenta el análisis de los parámetros de aplicación del UST en la literatura revisada.

\section{PARÁMETROS DE APLICACIÓN DEL UST EN LOS ESTUDIOS REVISADOS}

El UST es el resultado de la transducción de energía eléctrica en mecánica, produciendo vibraciones u ondas ultrasónicas cuya energía dependerá de los parámetros de aplicación como son la forma de generación del UST, la ERA, la frecuencia, la intensidad, el ciclo de trabajo que influencia la energía depositada en el caso de la forma de generación pulsada y el tiempo de tratamiento ${ }^{7}$. Los parámetros de aplicación de los estudios revisados y los resultados sobre la intensidad del dolor y la funcionalidad (variables de resultado principales en esta revisión) se encuentran resumidos en la tabla 3.

La forma de generación del UST puede ser continua o pulsada. El UST continuo se genera a través de la excitación constante del transductor con una onda sinusoidal de amplitud constante, produciendo una onda ultrasónica continua del 100\% del ciclo de trabajo ${ }^{25}$ es decir, que la intensidad y la energía generada por el UST se mantiene constante durante todo el tiempo de aplicación ${ }^{7}$. De los estudios revisados, seis aplicaron UST continuo ${ }^{14,15-19}$, de los cuales Mascarin et al. ${ }^{15}$, Luksurapan et al. ${ }^{16}$ y Kozanoglu et al. ${ }^{17}$ reportaron disminución de la intensidad del dolor en el grupo tratado.

Huang MH et al. ${ }^{13}$ y Loyola-Sánchez A et $\mathrm{al.}^{23}$, evaluaron el UST pulsado comparado con placebo, reportando aumento del rango de movimiento, aumento en la funcionalidad y disminución de la intensidad del dolor. Huang $\mathrm{MH}$ et $\mathrm{al}^{11}$, Cakir $\mathrm{S}$ et $\mathrm{al}^{12}$ y Tascioglu $\mathrm{F}$ et $\mathrm{al}^{20}$ evaluaron el UST continuo y pulsado ${ }^{11,12,20}$ mostrando diferencias significativas principalmente en los grupos tratados con UST pulsado excepto Cakir et al. ${ }^{12}$ quienes no encontraron diferencias significativas entre los grupos.

En cuanto al área de tratamiento, se ha propuesto que debe ser dos veces el tamaño de la ERA ${ }^{26}$, considerada el área del cabezal en el cual hay emisión efectiva de ondas acústicas ${ }^{7}$. El único estudio en reportar la ERA utilizada fue el de Loyola et al. ${ }^{23}$ aunque no es claro el área que reportan. Otros estudios reportan el diámetro del cabezal, información que no es suficiente para conocer el tamaño de la ERA, pues esta puede variar entre las marcas de equipos de UST.

Por otro lado, el UST producido por un transductor piezoeléctrico tiene un rango de frecuencia entre $1 \mathrm{y}$ 3.3 $\mathrm{MHz}^{7,8}$. Todos los estudios revisados utilizaron una frecuencia de $1 \mathrm{MHz}$, lo cual es justificado por la profundidad del tejido a tratar.

Uno de los parámetros que más variación muestra en los estudios que evalúan el efecto del UST en la OA es la intensidad, que se define como la tasa promedio de flujo de energía por unidad de área y es expresada en $\mathrm{W} / \mathrm{cm}^{2}$, indica la fuerza con que se suministra la energía al tejido en 1 segundo ${ }^{7,8}$. Así, el exceso de intensidad puede ser lesivo y la falta de intensidad puede no ser suficiente para alcanzar el objetivo terapéutico ${ }^{7,8}$.

La intensidad puede ser descrita en cuatro formas: la intensidad pico espacial pico temporal (SPTP), (Spatial Peak Temporal Peak), es la amplitud de la onda y viene definida por el fabricante del equipo; la intensidad pico espacial promedio temporal (SPTA), (Spatial Peak Temporal Average), describe la intensidad de energía que transcurre por la ERA del cabezal del UST, (se 
calcula dividiendo la potencia en (Watts) por la ERA en cm cuadrados $)^{8}$.

Los estudios reportan frecuentemente la intensidad pico temporal, (SATP) (Spatial Averaged Temporal Peak), para el UST continuo, la cual es definida como la SPTA durante el tiempo de emisión activa o "tiempo on". Para el caso de la intensidad del UST pulsado es aconsejable informar la intensidad promedio temporal, (SATA) (Average Temporal Averaged Spatial), resultante de multiplicar la SATP por el ciclo de trabajo ${ }^{8}$.

En los estudios revisados, la intensidad varió entre SATP 0.8 a 2 y SATA entre 0.3 a $2.5 \mathrm{~W} / \mathrm{cm}^{2}$, aunque este parámetro no es adecuadamente reportado ya que en algunos estudios no es claro cuál de las intensidades es la reportada, como el caso del estudio realizados por Loyola et al. ${ }^{23}$.

En cuanto al tiempo de tratamiento, es considerado el lapso durante el cual la zona es irradiada, e influye en la cantidad de energía que es aplicada al tejido. Sin embargo, el tiempo de tratamiento es una variable justificada con poca frecuencia en los estudios que aplican $\mathrm{UST}^{27}$. Oakley ${ }^{28}$, propuso aplicar UST durante uno o dos minutos en cada área de tratamiento correspondiente a tamaño y medio del cabezal, aunque esta relación no es justificada ${ }^{27,28}$. Algunos autores como Olsson et al. ${ }^{29}$ concluyen que no hay fórmula establecida para determinar el tiempo de tratamiento recomendado para el uso del UST.

Según los resultados obtenidos por Draper et al. ${ }^{26}$, cinco minutos de irradiación fueron suficientes para aumentar en $4^{\circ} \mathrm{C}$ la temperatura en los tejidos superficiales aplicando UST continuo, a una frecuencia de $3 \mathrm{MHz}$ y una intensidad de $1 \mathrm{~W} / \mathrm{cm}^{2}$. Los estudios que han aplicado UST en personas con OA, han utilizado tiempos de irradiación entre 3 y 12 minutos, la mayoría en un rango entre 3 y 5 minutos ${ }^{11,13,15,17,19,20}$ de aplicación en cada área de tratamiento aunque el argumento de tal elección no ha sido discutido en los estudios.

A partir de los parámetros anteriormente mencionados se determina la energía aplicada a cada zona de tratamiento, a través del siguiente cálculo ${ }^{30}$ :

$$
\text { Energía }(\mathrm{J})=\operatorname{SATA}\left(\mathrm{W} / \mathrm{cm}^{2}\right) * \operatorname{ERA}\left(\mathrm{cm}^{2}\right)^{*} \operatorname{Tiempo}(s)
$$

La energía expresada en julios (J), determina la cantidad de energía que recibe la zona de tratamiento ${ }^{31}$ siendo por tanto la mejor aproximación al concepto de dosis de aplicación del UST. Sin embargo, este concepto no es usualmente tenido en cuenta en la descripción de los parámetros en los estudios que evalúan el efecto del UST.

Además, los datos suministrados por los autores no son suficientes para realizar el cálculo de la energía aplicada en cada estudio, debido al reporte incompleto de parámetros como la ERA.

Teniendo en cuenta que el UST terapéutico es una modalidad física en cuyo caso se plantea que la energía aplicada es acumulable y además modificable según los parámetros de aplicación y el número de sesiones de tratamiento, el uso del concepto de energía como pieza clave al dosificar el UST debería ser considerado en estudios futuros con el objetivo de promover el acercamiento a la dosis necesaria para el tratamiento de pacientes con OA de rodilla.

Otro concepto fundamental en la dosificación del UST es la densidad energética, puesto que considera el área de tratamiento, lo cual es importante teniendo en cuenta que esta zona podría recibir variaciones en los niveles de energía debido a la velocidad, el recorrido de la aplicación y en si a la demarcación de la misma.

Densidad energética $\left(\mathrm{J} / \mathrm{cm}^{2}\right)=\frac{\operatorname{SATA}\left(\mathrm{W} / \mathrm{cm}^{2}\right) * \operatorname{ERA}\left(\mathrm{cm}^{2}\right) * \text { tiempo }(\mathrm{s})}{\text { Área de tratamiento }\left(\mathrm{cm}^{2}\right)}$

Teniendo en cuenta lo anterior, el concepto de densidad energética podría ser más indicado para determinar la dosis real de energía aplicada a cada $\mathrm{cm}^{2}$, sin embargo la importancia de estos conceptos no fue evaluada ni valorada en la literatura científica revisada.

A pesar de las limitaciones ya descritas, el análisis de la literatura revisada sugiere que el UST pulsado aplicado a $1 \mathrm{MHz}$, durante 3-5 minutos con una SATA entre 0.4 y $0.6 \mathrm{~W} / \mathrm{cm}^{2}$ (tabla 3 ) podría tener efectos positivos sobre la intensidad del dolor y la funcionalidad en personas con OA de rodilla, debido a los efectos mecánicos que serían alcanzados con niveles de intensidad baja. Esta ventana terapéutica coincide con la propuesta por Robertson $\mathrm{V}^{31}$ para el manejo del dolor en patología musculoesquelética.

Es necesario que ECC evalúen el efecto del UST sobre la intensidad del dolor, la movilidad articular, la fuerza muscular, la funcionalidad y la calidad de vida en personas con OA de rodilla, aplicando los parámetros descritos e incluyendo instrumentos de evaluación con buenas propiedades psicométricas, así como la evaluación de la influencia de covariables lo que podría reducir la introducción de sesgos en los resultados. 
TABLA 3. Parámetros de aplicación del UST y resultados sobre la intensidad del dolor y la funcionalidad en personas con OA de rodilla.

\begin{tabular}{|c|c|c|c|c|c|c|c|c|c|c|}
\hline \multirow[t]{2}{*}{ Autor } & \multicolumn{6}{|c|}{ Parámetros de aplicación del UST } & \multicolumn{2}{|c|}{$\begin{array}{c}\text { Intensidad del dolor } \\
(\text { EAV) }\end{array}$} & \multicolumn{2}{|c|}{ Funcionalidad } \\
\hline & Generación & $\begin{array}{l}\text { Frecuencia } \\
(\mathrm{MHz})\end{array}$ & $\begin{array}{c}\text { Intensidad } \\
\left(\mathrm{W} / \mathrm{cm}^{2}\right)\end{array}$ & $\begin{array}{l}\text { ERA } \\
\left(\mathrm{cm}^{2}\right)\end{array}$ & $\begin{array}{c}\text { TA } \\
\text { (minutos) } \\
\text { y sesiones }\end{array}$ & $\begin{array}{c}\text { Número } \\
\text { de } \\
\text { sesiones }\end{array}$ & $\begin{array}{l}\text { Línea de } \\
\text { base }\end{array}$ & $\begin{array}{l}\text { Finalizada la } \\
\text { intervención }\end{array}$ & $\begin{array}{l}\text { Línea de } \\
\text { base }\end{array}$ & $\begin{array}{c}\text { Finalizada } \\
\text { la } \\
\text { intervención }\end{array}$ \\
\hline $\begin{array}{l}\text { Mascarin N } \\
\text { et al. }\end{array}$ & Continuo & $1 \mathrm{MHz}$ & SATP 0.8 & NR & $3-4$ & 24 & $10.1 \pm 3.8 *$ & $6.2 \pm 4.2$ & $318 \pm 68 * *$ & $358 \pm 77$ \\
\hline \multirow[t]{2}{*}{$\begin{array}{l}\text { Özgönenel } \\
\text { et al }\end{array}$} & Continuo & $1 \mathrm{MHz}$ & SATP 1 & NR & 5 & 10 & $6.7 \pm 1.8$ & $3.9 \pm 2.0$ & $40.9 \pm 13.5 \dagger$ & $35.5 \pm 6.7$ \\
\hline & Placebo & & & & & & $5.1 \pm 2.3$ & $4.0 \pm 2.6$ & $40.9 \pm 9.8$ & $36.4 \pm 7.6$ \\
\hline \multirow[t]{2}{*}{ Ulus Y et al } & Continuo & $1 \mathrm{MHz}$ & SATP 1 & NR & 10 & 15 & $6.1 \pm 1.6$ & $3.30 \pm 1.83$ & $77.9 \pm 48.4 \dagger$ & $68.0 \pm 44.53$ \\
\hline & Placebo & $1 \mathrm{MHz}$ & & & & & $7.2 \pm 2.0$ & $4.45 \pm 2.16$ & $57.2 \pm 18.9$ & $51.2 \pm 15.74$ \\
\hline $\begin{array}{l}\text { Huang M } \\
\text { et al }\end{array}$ & Pulsado & $1 \mathrm{MHz}$ & SATA 0.6 & NR & 5 & 24 & $5.5 \pm 1.7$ & $3.0 \pm 1.8$ & $71.3 \pm 6.7 \dagger$ & $90.2 \pm 3.1$ \\
\hline $\begin{array}{l}\text { Loyola A } \\
\text { et al }\end{array}$ & Pulsado & $1 \mathrm{MHz}$ & N.R & $3.5-5$ & 9.5 & 24 & $2.6 \pm 2.5$ & $1.6 \pm 2.0$ & $36.8 \pm 11.8^{* *}$ & $47.7 \pm 13.4$ \\
\hline \multirow[t]{2}{*}{$\begin{array}{l}\text { Huang M } \\
\quad \text { et al }\end{array}$} & Continuo & $1 \mathrm{MHz}$ & SATP 1.5 & NR & 5 & 24 & $5.2 \pm 1.7$ & $3.3 \pm 0.8$ & $72.6 \pm 6.5 \dagger$ & $90.9 \pm 4.1$ \\
\hline & Pulsado & $1 \mathrm{MHz}$ & SATA 0.6 & NR & 5 & 24 & $5.0 \pm 1.3$ & $2.6 \pm 1.7$ & $73.2 \pm 6.0$ & $92.4 \pm 3.4$ \\
\hline \multirow[t]{2}{*}{$\begin{array}{l}\text { Tascioglu } \\
\text { et al }\end{array}$} & Continuo & $1 \mathrm{MHz}$ & SATP 2 & NR & 5 & 10 & $6.7 \pm 1.4$ & $5.22 \pm 1.7$ & $23.07 \pm 2.9 \ddagger$ & $22.85 \pm 2.9$ \\
\hline & Pulsado & $1 \mathrm{MHz}$ & SATA 0.5 & NR & 5 & 10 & $6.9 \pm 1.4$ & $5.25 \pm 1.9$ & $22.57 \pm 2.1$ & $20.00 \pm 1.9$ \\
\hline Cetin et al & Continuo & $1 \mathrm{MHz}$ & SATP 1.5 & NR & 10 & 24 & $5.9 \pm 1.4$ & $3.55 \pm 1.41$ & $53.10 \pm 9.8 \dagger$ & $42.60 \pm 11.5$ \\
\hline \multirow[t]{2}{*}{ Cakir et al } & Continuo & $1 \mathrm{MHz}$ & SATP 1 & NR & 12 & 24 & $57.9 \pm 20.2$ & $28.3 \pm 18.2$ & $\mathrm{NE}$ & $\mathrm{NE}$ \\
\hline & Pulsado & $1 \mathrm{MHz}$ & SATA 0.25 & NR & 12 & 24 & $55.7 \pm 17.8$ & $26.9 \pm 19.5$ & $\mathrm{NE}$ & $\mathrm{NE}$ \\
\hline $\begin{array}{l}\text { Luksurapan } \\
\text { et al }\end{array}$ & Continuo & $1 \mathrm{MHz}$ & SATP 1 & NR & 10 & 10 & $72.5 \pm 12.3$ & $43.91 \pm 25.19$ & $\mathrm{NE}$ & $\mathrm{NE}$ \\
\hline $\begin{array}{l}\text { Kozanoglu } \\
\text { et al }\end{array}$ & Continuo & $1 \mathrm{MHz}$ & SATP 1 & NR & 5 & 10 & $42.6 \pm 20.1$ & $27.7 \pm 17.1$ & $21.0 \pm 9.5 \ddagger$ & $11.8 \pm 2.0$ \\
\hline Boyaci et al & Continuo & $1 \mathrm{MHz}$ & SATP 1.5 & NR & 8 & 10 & $7.7 \pm 2.1$ & $5.7 \pm 1.9$ & $22.9 \pm 4.8 \bullet$ & $20.30 \pm 4.4$ \\
\hline $\begin{array}{l}\text { Oktayoğlu P } \\
\text { et al }\end{array}$ & Continuo & $1 \mathrm{MHz}$ & SATP 1.5 & NR & 10 & 10 & $61 \pm 13.7$ & $50 \pm 11.2$ & $11.8 \pm 4.33 ¥$ & $10.8 \pm 4.25$ \\
\hline
\end{tabular}

*Promedio \pm DE: promedio \pm desviación estándar; **Test de marcha de seis minutos; $\uparrow$ Test de 50 metros; $¥$ Test de 20 metros; • Test de 15 metros; ¥Escala funcional de Lequesne; SATP: Spatial Averaged Temporal Peak; SATA: Spatial Average Temporal Average; NR: No reportado; ERA: área de irradiación; TA: Tiempo de aplicación efectiva; EVA: Escala análoga visual; NE: No evaluado. Todos los resultados fueron estadísticamente significativos.

\section{EFECTOS FISIOLÓGICOS Y TERAPÉUTICOS DEL UST EN PERSONAS CON OA DE RODILLA}

Hasta la fecha no existen intervenciones terapéuticas de probada eficacia para detener la progresión del deterioro del cartílago articular en personas con OA de rodilla, siendo el dolor y la funcionalidad las principales dificultades percibidas por las personas con esta condición, las cuales afectan la calidad de vida y para los profesionales del área de salud son objetivo principal en el tratamiento ${ }^{32}$. La literatura revisada sugiera que el UST pulsado disminuye la intensidad del dolor y aumenta la funcionalidad en personas con OA de rodilla ${ }^{11,13,20,23}$.

Los mecanismos por los cuales el UST pulsado podría disminuir la intensidad del dolor, se relacionan con el efecto de la intervención sobre la disminución de la función de la bomba de sodio y potasio en las fibras de conducción dolorosa; el aumento en la permeabilidad de la membrana favoreciendo el aumento de la vascularización en los tejidos en reparación; la disminución de la óxido nítrico sintetasa y la activación de la compuerta del dolor (figura 2). A continuación se ampliarán los posibles mecanismos antes citados. 
La primera hipótesis que podría explicar el efecto del UST sobre la intensidad del dolor, es la disminución de la ATPasa reduciendo la función de la bomba de sodio y potasio en las membranas plasmáticas neuronales, afectando posiblemente la transducción de estímulos nociceptivos y la transmisión neural posterior, lo que podría dificultar la despolarización de las fibras nerviosas amielínicas $\mathrm{C}$ y delgadas mielínicas $a \delta^{33}$, disminuyendo la conducción del dolor en estas fibras, dando lugar a la disminución de la intensidad del dolor ${ }^{33}$.

Dinno et al. ${ }^{33}$ evaluaron los cambios electrofisiológicos en las membranas celulares a través de la alteración del potencial eléctrico en las células epidérmicas de una rana tras la aplicación de UST pulsado y continuo a $1 \mathrm{MHz}$ y una SATA entre 0.06 y $0.45 \mathrm{~W} /$ $\mathrm{cm}^{2}$, reportando disminución en un $50 \%$ del potencial eléctrico y de la resistencia en las células tratadas, además del aumento de la conductancia iónica lo que sugiere alteración de la fuerza electromotriz de la bomba de sodio y potasio se altera.

También reportaron que el UST pulsado fue más efectivo que el continuo en generar estos cambios electrofisiológicos. La disminución de la fuerza electromotriz de la bomba puede ser dada por la disminución de la enzima ATPasa, responsable de producir hidrólisis del ATP necesario para el funcionamiento de la bomba, esto explicaría la disminución en la salida de potasio que también fue reportada y podría favorecer la disminución del dolor ${ }^{33}$.
Otros autores han mencionado que es posible que la alteración de la bomba de sodio y potasio como fue descrita pueda suceder en las fibras A delta y C alterando la transmisión del dolor ${ }^{34}$. Sin embargo, no fueron encontrados en la literatura revisada estudios que evalúen la disminución o el bloqueo de la velocidad de conducción nerviosa en estas fibras luego de la aplicación de UST, por el contrario ha sido reportado un aumento en la velocidad de conducción nerviosa de las fibras A beta, cuyo incremento es asociado en estos estudio al aumento de la temperatura ${ }^{26}$.

Por otro lado, el aumento de la permeabilidad de la membrana también se asocia a factores que pudieran favorecer la reparación del condrocito y la MEC en seres humanos ${ }^{35}$. Estudios in vitro han mostrado que los fenómenos biofísicos del UST pulsado como la cavitación y las microcorrientes acústicas podrían cambiar la permeabilidad tisular favoreciendo el proceso de reparación ${ }^{35}$.

En relación a lo anterior, Dinno et al..$^{33}$ sugieren que los cambios celulares, tales como alteraciones en la motilidad y la estimulación de la síntesis, inducidas por intensidades bajas de UST (SATA entre 0.1 y $0.5 \mathrm{~W} /$ $\mathrm{cm}^{2}$ ) se deben principalmente a los efectos no térmicos, además estos procesos están asociados a los cambios en la permeabilidad de la membrana celular y al transporte de iones y moléculas a través de ella ${ }^{33}$.

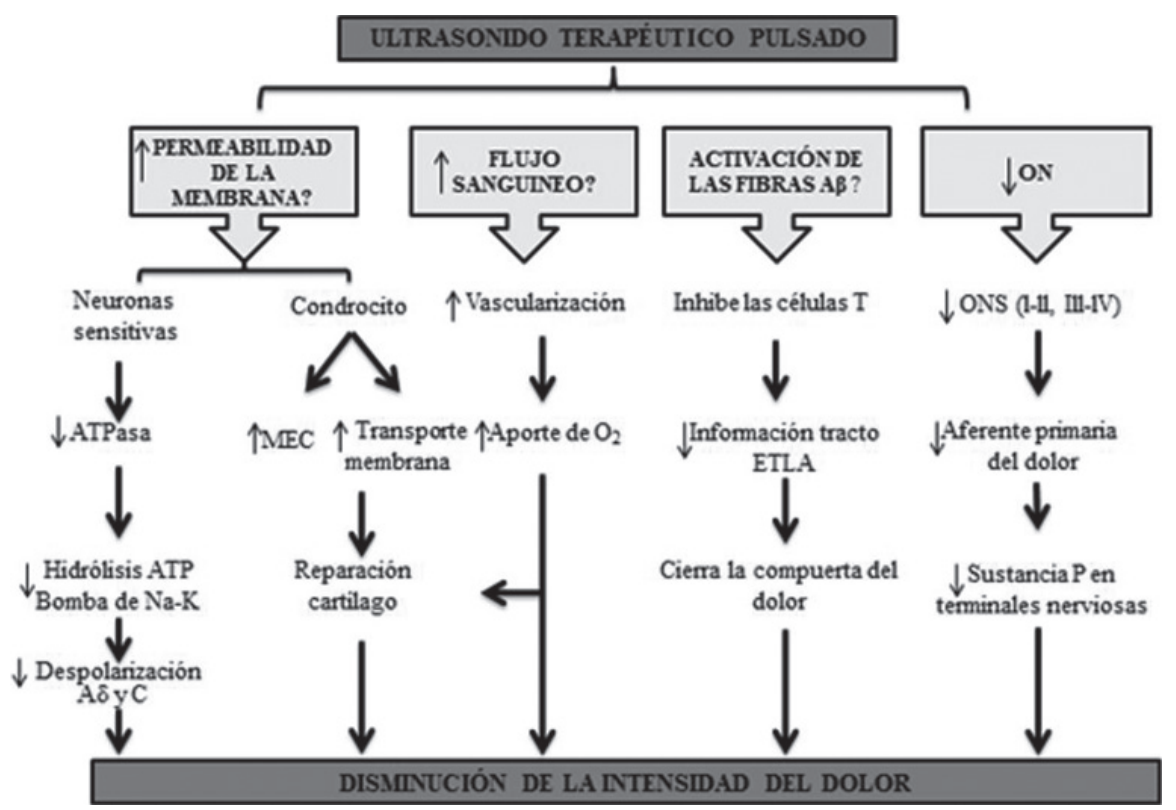

FIGURA 2. Mecanismos fisiológicos por los cuales el UST podría disminuir la intensidad del dolor en personas con OA de rodilla. 
En los tejidos epiteliales in vivo ${ }^{34} \mathrm{e}$ in vitro ${ }^{35}$, se ha demostrado que el UST puede influenciar las vías de transporte de la membrana celular y paracelular o vías intercelulares, puesto que la recepción y transmisión de señales ambientales en la membrana podrían estar implicadas en la determinación del destino celular favoreciendo la actividad de segundos mensajeros, ocasionando alteraciones en la expresión génica, diferenciación y la adquisición de características especiales por las células estimuladas ${ }^{36}$, lo que puede tener valor terapéutico en los tejidos en reparación.

Otro elemento importante a favor de la hipótesis de la reparación del tejido periarticular y la disminución del dolor, es un posible aumento de la vascularización de la zona tratada, dado que el incremento del flujo sanguíneo en los tejidos blandos promueva la liberación de factores de crecimiento y citokinas que favorezcan el proceso de reparación y aumenten el aporte de oxígeno que a su vez favorezca la disminución del dolor y la inflamación ${ }^{37}$. En este sentido, Rawool et al. ${ }^{37}$ mediante UST diagnóstico demostraron que el UST pulsado de baja intensidad aplicado durante 10 días favoreció el aumento significativo de la vascularización en la osteotomía de cúbito en perros, lo cual apoya esta hipótesis.

Otra posible explicación para el efecto del UST sobre el dolor, es la reducción de la óxido nítrico sintetasa (ONS) inducible en la médula espinal, reduciendo así la actividad del óxido nítrico en el procesamiento del dolor y la inflamación ${ }^{38}$. El óxido nítrico $(\mathrm{ON})$ es sintetizado por la enzima ONS y es la primera sustancia química gaseosa producida por células vivas para enviar señales intercelulares, actuando como mediador biológico $^{38}$. Además, se cree que el ON puede participar directamente en la regulación de la transmisión sináptica en la sustancia $\mathrm{P}$ contenida en las terminales nerviosas mediando así la transmisión aferente primaria del dolor ${ }^{38}$.

Yueh-Ling ${ }^{38}$ evaluó el efecto del UST sobre la ONS a nivel espinal en las láminas I-II, III-IV, V-VI del asta dorsal en ratas sometidas a OA inducida por coadyuvante de Freund, encontrando que el UST pulsado a una SATA de $0.5 \mathrm{~W} / \mathrm{cm}^{2}, 1 \mathrm{MHz}$ y un tiempo de aplicación de 5 minutos disminuyó la ONS en las mismas láminas, tanto en el grupo de fonoforesis con diclofenaco como en el grupo de UST en comparación al grupo placebo.

Por último, la teoría de la compuerta del dolor desarrollada por Melzack y Wall podría apoyar también los efectos del UST sobre la intensidad del dolor. Esta teoría sugiere la existencia de un sistema que puede limitar la información de las vías del dolor a nivel central, en este sentido las fibras A delta y $\mathrm{C}$ facilitan la transmisión abriendo la compuerta del dolor, mientras que las fibras A alfa y beta pueden limitar la transmisión cerrando compuerta ${ }^{39}$. Con la aplicación del UST, el estímulo generado por el contacto del cabezal del UST podría cerrar la compuerta al activarse un receptor cutáneo mediante la vibración o el tacto, cuya información es trasmitida por las fibras gruesas $\mathrm{A} \beta$ (fibras rápidas), las cuales inhiben las células de transmisión disminuyendo la información dolorosa conducida por el tracto espinotalámico ascendente cerrando la compuerta del dolor ${ }^{39}$.

Adicionalmente, el tracto espinotalámico descendente podría modular la información dolorosa liberando péptidos opiáceos endógenos, como encefalinas y dinorfinas que facilitan la disminución de la intensidad dolorosa, sin embargo en la literatura revisada no fue encontrada evidencia científica que soporte esto con el uso del UST ${ }^{39}$.

Por otro lado, es posible que la mayor absorción de la energía del UST en los tejidos tratados haya favorecido la disminución de la intensidad del dolor, teniendo en cuenta que el coeficiente de absorción de la energía del UST es mayor en los tejidos con gran contenido de colágeno como los que fueron blanco de las aplicación en los estudios revisados (tendón de la pata de ganso, inserción de la fascia lata, articulación patelofemoral y

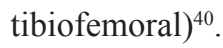

Binder et $\mathrm{al}^{40}$ aplicaron UST pulsado con los mismos parámetros durante 10 minutos en el tratamiento de la epicondilitis, reportando disminución significativa de la intensidad del dolor en reposo y al movimiento, luego de 12 sesiones de aplicación. Estos resultados apoyan los efectos terapéuticos sobre la intensidad del dolor con la aplicación de UST pulsado de baja intensidad en el tratamiento de lesiones músculo esqueléticas crónicas en zonas con alto contenido de colágeno como la OA en la rodilla.

\section{CONCLUSIÓN}

El UST pulsado parece ser efectivo en la disminución de la intensidad del dolor y en aumentar la funcionalidad en personas con OA de rodilla aplicado a $1 \mathrm{MHz}$, durante 3-5 minutos con una SATA entre $0.4 \mathrm{y}$ $0.6 \mathrm{~W} / \mathrm{cm}^{2}$. Sin embargo, estos resultados están soportados en estudios con limitaciones metodológicas 
y de difícil comparación. Se hace necesario confirmar la eficacia del UST mediante ECC donde se evalúen los efectos a largo plazo de estas intervenciones y los efectos de las mismas sobre la reparación del cartílago.

Por otro lado, los mecanismos por los cuales el UST reduce la intensidad del dolor en la OA de rodilla deben ser estudiados a profundidad para comprender los mediadores moleculares potencialmente involucrados en la respuesta terapéutica observada.

\section{AGRADECIMIENTOS}

Escuela de Fisioterapia, Universidad Industrial de Santander.

\section{REFERENCIAS BIBLIOGRÁFICAS}

1. Zhang W, Moskowitz RW, Nuki G, Abramson S, Altman RD, Arden N, etal. OARSI recommendations for the management of hip and knee osteoarthritis, Part II:OARSI evidence-based, expert consensus guidelines. Osteoarthritis Cartilage. 2008; 16(2): 137-162.

2. Silverwood V, Blagojevic BM, Jinks C, Jordan JL, Protheroe J, Jordan KP. Current evidence on risk factors for knee osteoarthritis in older adults: a systematic review and meta-analysis. Osteoarthritis Cartilage. 2015; 23(4): 507-515.

3. Peñaloza RE, Salamanca N, Rodríguez JM, Rodríguez J, Beltrán AR. Estimación de la carga de enfermedad para Colombia, 2010. 1ed. Bogota: Editorial Pontificia Universidad Javeriana; 2014.

4. Hochberg MC, Altman RD, April KT, Benkhalti M, Guyatt G, McGowan J. American college of rheumatology 2012 recommendations for the use of nonpharmacologic and pharmacologic therapies in osteoarthritis of the hand, hip, and knee. Arthritis Care Res. 2012; 64(4): 465-474.

5. Jamtvedt G, Dahm KT, Christie A, Moe RH, Haavardsholm E, Holm I, et al. Physical therapy for patients with osteoarthritis of the knee, an overview of systematic reviews. Phys Ther. 2008; 88(1): 123-136.

6. Loyola-Sánchez A, Richardson J, Macintyre NJ. Efficacy of ultrasound therapy for the management of knee osteoarthritis: a systematic review with meta-analysis. Osteoarthritis Cartilage. 2010; 18(8): 1117-1126.

7. Rice D, McNair P. Quadriceps arthrogenic muscle inhibition: Neural mechanisms and treatment perspectives. Semin arthritis rheum. 2010; 40(30): 250-266.
8. Cameron M. Ultrasound. In: Cameron M. Physical Agents in Rehabilitation: From Research to Practice. 4th ed. Philadelphia: WB. Saunders; 2003: p.173-198.

9. Dreinhöfer K, Stucki G, Ewert T, Huber E, Ebenbichler G, Gutenbrunner C, et al. ICF Core Sets for osteoarthritis. J Rehabil Med. 2004; 44: 75-80.

10. Cibulka MT, White DM, Woehrle J, Harris Hayes M, Enseki K, Fagerson TL, et al. Hip pain and mobility deficits hip osteoarthritis: clinical practice guidelines linked to the international classification of functioning, disability, and health from the orthopaedic section of the American Physical Therapy Association. J Orthop Sports Phys Ther. 2009; 39(4): 1-25.

11. Huang MH, Lin YS, Lee CL, Yang RC. Use of Ultrasound to Increase Effectiveness of Isokinetic Exercise for Knee Osteoarthritis. Arch Phys Med Rehabil. 2005; 86(8): 345-352.

12. Cakir S, Hepguler S, Ozturk C, Korkmaz M, Isleten B, Atamaz FC. Efficacy of therapeutic ultrasound for the management of knee osteoarthritis: a randomized, controlled, and double-blind study. Am J Phys Med Rehabil. 2014; 93(5): 405-412

13. Huang MH, Yang RC, Lee CL, Chen TW, Wang MC. Preliminary results of Integrated Therapy for Patients With Knee Osteoarthritis. Arthritis \& Rheumatism 2005; 53(6): 812-820.

14. Cetin N, Aytar A, Atalay A, Akman MN. Comparing Hot Pack, Short-Wave Diathermy, Ultrasound, and TENS onIsokinetic Strength, Pain, and Functional Status of Women with Osteoarthritic Knees. Arm. J. Phys. Med. Rehabil. 2008; 87(6): 443-451.

15. Mascarin NC, Vancini RL, Andrade ML, Magalhães Ede P, de Lira CA, Coimbra IB. Effects of kinesiotherapy, ultrasound and electrotherapy in management of bilateral knee osteoarthritis: prospective clinical trial. BMC Musculoskeletal Disorders. 2012; 13: 182-190.

16. Luksurapan W, Boonhong J. Effects of Phonophoresis of Piroxicam and Ultrasound on Symptomatic Knee Osteoarthritis. Arch Phys Med Rehabil. 2013; 94(2): 250-255.

17. Kozanoglu E, Basaran S, Guzel R, Guler Uysal F. Short term efficacy of ibuprofen phonophoresis versus continuous ultrasound therapy in knee osteoarthritis. Swiss Med Wkly. 2003; 133(23): 333-338.

18. Ulus Y, Tander B, Akyol Y, Durmus D, Buyukakıncak $\mathrm{O}$, Gul U, et al. Therapeutic ultrasound versus sham ultrasound for the management of patients with knee osteoarthritis: a randomized double-blind controlled 
clinical study. Int J Rheum Dis. 2012; 15(2): 197-206.

19. Ozgönenel L, Aytekin E, Durmuşoglu G. A doubleblind trial of clinical effects of therapeutic ultrasound in knee. Ultrasound Med Biol. 2009; 35(1): 44-49.

20. Tascioglu F, Kuzgun S, Armagan S, Ogutler G. Short-term Effectiveness of Ultrasound Therapy in Knee Osteoarthritis. J Int Med Res. 2010; 38(4): 1233-1242.

21. Boyaci A, Tutoglu A, Boyaci N, Aridici R, Koca I. Comparison of the efficacy of ketoprofen phonophoresis, ultrasound, and short-wave diathermy in knee osteoarthritis. Rheumatol Int. 2013; 33(11): 2811-2818.

22. Oktayoğlu P, Gür A, Yardımeden I, Çağlayan M, Çevik F, Bozkurt M, et al. Comparison of the Efficacy of Phonophoresis and Conventional Ultrasound Therapy in Patients with Primary Knee Osteoarthritis. Erciyes Med J. 2014; 36: 11-18.

23. Loyola-Sánchez A, Richardson J, Beattie KA, Otero C, Adachi JD, MacIntyre NJ. Effect of Low-Intensity Pulsed Ultrasound on the Cartilage Repair in People With Mild to Moderate Knee Osteoarthritis: A Double-Blinded, Randomized, Placebo-Controlled Pilot Study. Arch Phys Med Rehabil. 2012; 93(1): 35-42.

24. Olivo SA, Macedo LG, Gadotti IC, Fuentes J, Stanton T, Magee DJ. Scales to assess the quality of randomized controlled trials: A systematic review. Physioth. 2008; 88(2): 156-175.

25. Humphrey VF. Review: Ultrasound and matter -Physcial interactions. Prog Biophys mol biol. 2007; 93(1): 195-211.

26. Draper DO, Castel JC, Castel D. Rate of Temperature Increase in Human Muscle During $1 \mathrm{MHz}$ and 3 $\mathrm{MHz}$ Continuous Ultrasound. J Orthop Sports Phys Ther. 1995; 22(4): 143-150.

27. Farcic TS, Baldan CS, Cattapan CG, Parizotto NA, João SM, Casarotto RA. Treatment time of ultrasound therapy interferes with the organization of collagen fibers in rat tendons. Braz J Phys Ther. 2013; 17(3): 263-271.

28. Oakley EM. Application of continuous beam ultrasound at therapeutic levels. Physiotherapy. 1978; 64(6): 169-172.
29. Olsson DC, Martins MV, Pippi NL, Mazzanti A, Tognoli GK. Ultra-som terapêutico na cicatrização tecidual. Ciênc Rural. 2008; 38: 1199-1207.

30. Rodriguez M. Ultrasonidos. En: Rodriguez M. Electroterapia en fisioterapia. 2da ed. Buenos Aires: Editorial médica panamericana. 2004: p. 515-551.

31. Robertson VJ. Dosage and treatment response in randomized clinical trials of therapeutic ultrasound. Phys Ther Sport. 2002; 3: 124-133.

32. Dreinhöfer K, Stucki G, Ewert T, Huber E, Ebenbichler G, Gutenbrunner C, et al. ICF Core Sets for osteoarthritis. J Rehabil Med. 2004; 44: 75-80.

33. Dinno MA, Dyson M, Young SR, Mortimer AJ, Hart J, Crum LA. The significance of membrane changes in the safe and effective use of therapeutic and diagnostic ultrasound. Phys Med Biol. 1989; 34(11): 1543-1552.

34. Mardiman S, Wessel J, Fisher B. The effect of ultrasound on the mechanical pain threshold of healthy subjects. Physiotherapy. 1995; 81: 718-723.

35. Tedesco LS, Fuseler J, Grisham M, Wolf R, Roerig SC. Therapeutic administration of nitric oxide synthase inhibitors reverses hyperalgesia but not inflammation in a rat model of polyarthritis. Pain. 2002; 95(3): 215-223.

36. Miyasaka N, Hirata Y. Nitric oxide and inflammatory arthritides. Life Sci. 1997; 61(21): 2073-2081.

37. Rawool NM, Goldberg BB, Forsberg F, Winder AA, Hume E. Power Doppler assessment of vascular changes during fracture treatment with low-intensity ultrasound. J Ultrasound Med. 2003; 22(2): 145-153.

38. Hsieh YL. Effects of ultrasound and diclofenac phonophoresis on inflammatory pain relief: suppression of inducible nitric oxide synthase in arthritic rats. Phys Ther. 2006; 86(1): 39-49.

39. Summers S. Evidence-based practice part 1: pain definitions pathophysilogic mechanisms and theories. J Perianeth Nur. 2000; 15(5): 357-365.

40. Binder A, Hodge G, Greenwood AM, Hazleman BL, Page Thomas DP. Is therapeutic ultrasound effective in treating soft tissue lesions?. Br Med J (Clin Res Ed). 1985; 290(6467): 512-514. 